\section{Die nachhaltige Fakultät}

\section{E. Taverna}

\section{Liebe Kolleginnen, liebe Kollegen}

Sie haben Post von der Fakultät erhalten, viele Jahre nach dem Staatsexamen, für manche ein ganzes Leben später. Fast alles hat sich seither verändert, nur eines nicht: die Fakultät schickt ihnen einen Einzahlungsschein. 390 Gründungsmitgliedern ist der neue Verein der Alumni ein Beitrag wert. Was lockt uns in diesen verdunkelten Hörsaal mit seinem Sitzkomfort, der an die industrielle Tierhaltung erinnert? Sind es die paar Lateinbrocken auf der Einladung, die einst wie geknickte Federn zum Klinikschmuck unserer Häuptlinge gehörten? Schmeichelt mich das späte Interesse des Dekanats? Oder ist es der Zweckartikel der neuen Statuten, der drei Ziele erwähnt: die Förderung persönlicher Kontakte, den Wissensaustausch und die Unterstützung von Projekten?

Dass ich hier als gewöhnlicher Dorfarzt reden darf oder muss, verdanke ich meinem Bericht über die erste Versammlung vor einem Jahr, der in unserem gelben Hausblatt abgedruckt war. Wie früher im Militärdienst, so vermute ich wenigstens, wird der Bock zum Gärtner gemacht, auf dass er mit seiner Kritik bei der nächsten Wortmeldung vorsichtiger umgehe.

«Wie nachhaltig wirkt die Fakultät?» Eine beliebte Leerformel für eine ungewohnte Frage. Sind wir spät Gerufene Gegenstand eines Evaluationsverfahrens, etwa im Rahmen neuer Qualitätsprüfungen, müssen wir das Staatsexamen wiederholen? Der alte Begriff aus der Forstwirtschaft meint heute eine dauerhafte, umwelt-, sozial- und wirtschaftsverträgliche $\mathrm{Zu}$ kunftsgestaltung. Soll ich die medizinische Fakultät, dreissig Jahre später, an diesen Kriterien messen? Hat die damalige Ausbildung dazu beigetragen, dass sie und ich uns umwelt-, sozial- und wirtschaftsverträglich verhalten haben?

Meine zwischenzeitliche Existenz hat sich beruflich, akademiefern, an der Basis abgespielt. Die Gravitationszentren meiner Weiter- und Fortbildung waren und sind Kantons- und Bezirksspitäler, von einer Assistentenzeit in Neuseeland abgesehen. Wer sich auf dem Lande niederlässt, richtet sich im Alltag nach seinem Hausspital, wo mit zeitlicher Verzögerung die Lernkaskaden und Erschütterungen aus den universitären Epizentren wirksam werden.

Gestatten sie mir eine kurze Nabelschau, bevor ich zum Allgemeinen übergehe. In meine Studienjahre in Zürich fallen die Erfindung des Minijupe, der Globuskrawall und die Rede eines Anhängers von Rudi Dutschke im überfüllten, grossen Hörsaal der ETH. Die 68er Unruhen gingen an unserem Semester fast spurlos vorüber, von einer kleinen Gruppe Idealisten abgesehen, die von sich aus Reformvorschläge erar- beitete, die kürzlich ein internationales Expertenteam, zumindest teilweise, wiederholt hat. Wir hatten wichtigeres zu tun, als die Gesellschaft zu verändern. Wir mussten eine Karriere vorbereiten, für Prüfungen schanzen, militärische Grade abverdienen, in überfüllten Hörsälen einen Platz ergattern und im langjährigen Frontalunterricht, didaktisch auch mässig Vorgetragenes mitschreiben. Und doch hatte sich etwas unwiderruflich verändert, denn erstmals verstand ich mich als kleines Rädchen im real existierenden Getriebe.

Es gab wenig Manuskripte, dafür teure Lehrbücher, die mit Dozentenrabatt erhältlich das Schwänzen erleichterten. Die Frauen unter uns waren eine Minderheit, die das männliche Umfeld in den Pausen mit Rauchen kompensierte. Zu den wenigsten Lehrern bestand ein persönlicher Kontakt, meist nicht einmal im Examen. Wenn jemand im nächsten Semester nicht mehr erschien, durchgefallen, weggezogen oder gestorben, es war, als hätten er oder sie nie existiert. Von nachhaltiger Wirkung blieben für mich der Unterricht des sogenannten Mittelbaus im ansatzweise praktizierten Gruppenunterricht, motivierend waren das Praktikum und das "Repisemester» am Schluss. Die Vorklinik unterschied sich vorteilhaft durch einen strukturierten Lehrplan von den oft genug chaotisch wirkenden, klinischen Semestern. Ich war froh, der Fakultät zu entrinnen, den lichtlosen Sälen, dem permanenten Bauplatz, dem langen täglichen Sitzen über den unter sich zusammenhanglosen Vorträgen. Fächerübergreifenden Unterricht gab es erst am Ende und Blockunterricht blieb ein Projekt. Dafür war es noch leicht möglich, die Uni zu wechseln, ein Auslandsemester zu integrieren oder fachfremde Vorlesungen anzuhören.

Die Weiterbildung dauerte länger als das Studium, die Wirkung der praktischen Lehrmeister war in jeder Hinsicht nachhaltiger. Einmal niedergelassen, hatte ich Jahr für Jahr mit Studenten zu tun, die sich in unsere ländliche Gemeinschaftspraxis getrauten. Damit blieb eine dünne Nabelschnur mit den Fakultäten aus Basel, Bern und Zürich bestehen. Es war spürbar, dass die Zürcher auf Forschung und die Berner auf Didaktik setzten. Es war auch interessant, wie diese jungen Menschen nicht nur neue Lehrinhalte sondern auch neue Weltsichten zu uns brachten. Sie erschienen mir oft pragmatischer, zielstrebiger, wissender und besser auf die Patienten vorbereitet, aber auch zunehmend im Lernstress und eingeengt durch eine unpersönliche Schulmechanik. Die Berner schneiden in meiner Erinnerung besser ab, vielleicht weil ihr Unterricht, dank dem eigens eingeführten, pädagogischen Institut von Professor Pauli, dem Zürcher Modell überlegen war. Ein geplantes Klinikum am Kantonsspital St. Gallen, das Zürich entlastet hätte, stiess hier auf wenig Gegenliebe. Das Kartell der Machtträger funktionierte auch später, wenn es galt, Eindringlinge wie die Allgemein- oder die Komplementärmedizin zu neutralisieren.

Immer noch wollen trotz Aufnahmeprüfung mehr Maturanden Medizin studieren als Plätze frei sind, selbst wenn es an Ingenieuren und Lehrern mangelt. 
Seit meiner Gymnasialzeit wird an den Mittelschulen viel von Berufswahl geredet, aber wenig getan. Die Art, wie die medizinische Fakultät mit Interessierten umging, fand ich absolut schockierend. Meine ältere Tochter wollte zuerst Medizin studieren. Das versprach 1994 einen zeitraubenden, administrativen Hürdenlauf, jede Menge Formulare, arrogante Auskünfte und Ungewissheit über die zugewiesene Fakultät bis eine Woche vor Semesterbeginn, damit ohne Möglichkeit, rechtzeitig für eine Unterkunft zu sorgen. Gut motivierte, junge Menschen, das viel zitierte Humankapital, wurden wie lästige Bittsteller behandelt, die es möglichst fortzuscheuchen galt. Die Informationen aus den Zentren waren lückenhaft, widersprüchlich und oft schon durch Pressemitteilungen der folgenden Woche überholt. Es gab offensichtlich keine Absprache unter den Universitäten, denn jede Fakultät handelte im Alleingang, als gäbe es keine Hochschulkonferenzen. Und wiederum war es deprimierend, wie ein Bild mit Kommentar in der NZZ vom letzten Herbst den doppelten Maturandenjahrgang stehend und in überfüllten Räumen zeigte. Obwohl auch diese Probleme leicht vorauszusehen waren.

Im "MedInfo", einem vierseitigen Informationsblättchen der Fakultät Zürich, spricht der neue Dekan im Februar dieses Jahres vom zunehmend kompetitiven Umfeld, vom Fundraising und den mässig guten Noten, die eine internationale Expertengruppe, zwecks späterer Akkreditierung, austeilte. Vielleicht zeigt diese Kritik am Curriculum bereits Folgen, werden doch in der Schweizerischen Ärztezeitung Studienkoordinatoren und wissenschaftliche Mitarbeiter für Kreditpunktsysteme gesucht.

Das digitale Zeitalter bringt eine Dezentralisierung der Hochschulen, eine Verlagerung der Lehre in ein ganzes Netz von Anbietern. Vielleicht wird die Fakultät zur Filiale von Wirtschaftsunternehmen, vielleicht ist es mit dem Ausbildungsmonopol vorbei und die Hirslandengruppe oder andere öffentliche und private Fachhochschulen schnappen sich die besten Studienanwärter. Bereits zeichnet sich ab, dass die bisher einheitliche Ausbildung durch neue Studiengänge abgelöst wird, die für Nachdiplomstudien wie "Management im Gesundheitswesen" und einen völlig veränderten Gesundheitsbetrieb das Personal bereitstellen. Reformen wie Fleiner I und II, das neue Bundesgesetz über die Förderung der Universitäten und die Zusammenarbeit im Hochschulbereich, sowie der zunehmende Kostendruck, bauen auch die Universitäten radikal um, trotz des Widerwillens der meisten Kantone, ihre Kompetenzen abzugeben. Das Bundesamt für Statistik veröffentlichte im Dezember 2000 eine Erhebung über die universitären Lehr- und Forschungsaufwendungen in Spitälern. Innerbetrieblich werden 2\% für die Lehre und 9\% für die Forschung aufgewendet, total 720 Millionen Franken. Die Unterschiede zwischen den Spitälern werden, wen erstaunt es, als extrem gross bezeichnet. Dazu kommen die veränderten Bedürfnisse einer neuen Generation mit einem stetig zunehmenden Frauenanteil unter den Universitätsabsolventen. 1999 für Medizin und Pharmazie mit 54,5\%, an dritter Stelle, nach den Sozial- und Geisteswissenschaften. Berücksichtigt man noch die neuen Curricula und die Abgänge aller geplanten Fachhochschulen, dann wird das mit Sicherheit die immer noch patriarchal dominierte Medizinalkultur gründlich verändern.

Als nebenamtlicher Kantonsarzt habe ich Einsicht in die gesundheitspolitischen Planungen auf kantonaler und eidgenössischer Ebene. Ich sehe, wie zahlreiche Akteure mit- und vor allem gegeneinander arbeiten, wie gesellschaftliche Veränderungen und neue Gesundheitsberufe das traditionelle Rollenbild der Ärztinnen und Ärzte tiefgreifend umkrempeln. Ich erlebe mit ihnen, wie die FMH unter Druck gerät, wie sich neue Allianzen bilden und wie neue Technologien immer schneller die Halbzeit des eigenen Wissens verkürzen.

Was erwarte ich nach 30 Jahren Funkstille von der medizinischen Fakultät? Sie ist ja nicht mehr die Institution von damals, sie wird es immer weniger sein. In Erinnerung geblieben sind mir einige herausragende Lehrer. Auch bin ich immer noch interessiert an allen wissenschaftlichen Entwicklungen. Eine Alumniorganisation muss mehr bieten als ein Altherren- oder Altdamenkränzchen für vereinsmässige Nostalgiepflege, muss mehr bieten als einen Stammtisch für Prominentenklatsch, muss mehr sein als Sponsorenclub für fakultäre Gärtchenpflege oder Feigenblatt einer sogenannten "Öffnung".

Die Nachhaltigkeit der Ausbildungsjahre, fern vom Klinikbetrieb des Universitätsspitals mit seiner Spitzenmedizin, ist bescheiden ausgefallen. Und trotzdem, sie und ich suchen vielleicht etwas Gemeinsames. Immerhin beschäftige ich mich bereits mehr mit der Universität als alle die Jahre zuvor. Ich entdecke wieder die Studentenzeitungen und merke plötzlich, dass die Universitätsleitung ein interessantes, schön gestaltetes Magazin herausgibt, so zum Beispiel im April 2001 über «Nachhaltige Entwicklung». Selbst über das Internet lohnt sich allemal ein Mausklick. Es könnten sich ja neue Chancen auftun, neues Leben aus den Ruinen blühen. Etwa in Form von Orientierungen oder gar Wegweisern im unüberblickbaren Gesundheitsmarkt, von Denkhilfen zur Spitzenmedizin, zu umstrittenen Inhalten der Komplementärmedizin, zu Informationen aus der Welt der Forschung und des Unterrichts. Eine Öffnung nach aussen, die auch mich einschliesst, vielleicht sogar ein basisdemokratisches Mitspracherecht einräumt und damit zu verantwortlichem Mitdenken auffordert. Doch das ist, aller Voraussicht nach, total unrealistisch.

Meine Damen und Herren, ich danke ihnen für ihre Aufmerksamkeit. 